\title{
Drahtlose Anbindung kapazitiver Sensoren durch induktiv gekoppelte Felder
}

\author{
Th. Frank, St. Herbst, A. H. Khan, V. Kiran \\ CiS Forschungsinstitut für Mikrosensorik und Photovoltaik GmbH, Konrad-Zuse-Straße 14, \\ 99099 Erfurt
}

\begin{abstract}
Kurzfassung
Dieser Artikel beschreibt ein Verfahren zur drahtlosen Auswertung kapazitiver Sensoren durch induktiv gekoppelte Felder. Das System besteht aus einem LC-Schwingkreis, welcher einen kapazitiv arbeitenden Sensor enthält. Die Resonanzfrequenz ist abhängig von der variablen Kapazität und somit abhängig von der Messgröße. Über je eine Sende- und Empfangsspule wird der LC-Schwingkreis angeregt und die Resonanzfrequenz ausgewertet. Der LC-Schwingkreis benötigt keinerlei elektrische Zuführungen, er erhält die benötigte elektrische Energie über die induktive Kopplung der Sendespule mit der Induktivität des LC-Schwingkreises. Für den LC-Schwingkreis werden thermisch hoch belastbare Materialien verwendet, so dass das System für Temperaturen über $200^{\circ} \mathrm{C}$ geeignet ist. Untersucht wurden die Messgrößen Druck und Konzentration.
\end{abstract}

Schlüsselwörter: Drahtlos, kapazitiver Sensor, induktive Kopplung, Hochtemperatur, Druck, Konzentration

\section{Einleitung}

Eine Strategie zur Erhöhung der Material- und Energieeffizienz in der Produktion und im Alltag besteht in der Minimierung der Regelabweichung entscheidender Prozesse. Besonders in der chemischen Industrie kann durch eine optimale Prozessführung, sowohl der Umsatz erhöht, als auch der Energieverbrauch reduziert werden.

Von großer Bedeutung sind chemische Prozesse, welche bei hohen Temperaturen und hohem Druck ablaufen. Hier besteht ein besonders hohes Einsparungspotential durch eine optimierte Prozessführung. Um dynamische Vorgänge ortsaufgelöst darzustellen, wird zudem eine hohe Anzahl von Sensoren benötigt. Nur in gewissen Grenzen können Sensoren und Elektronik diesen Bedingungen direkt ausgesetzt werden. Dies liegt an der maximalen Einsatztemperatur elektronischer Schaltkreise und siliziumbasierter Sensoren. Hinzu kommt die chemische Beständigkeit der notwendigen Metallisierungen, Passivierungen und AVT-Materialen. Ein Einsatz oberhalb dieser Grenze führt zu einer überproportionalen Verteuerung, verstärkt durch die geforderte chemische Beständigkeit.

Der Artikel beschreibt den Aufbau und Funktion der hergestellten Prototypen eines drahtlosen, kapazitiven, induktiv gekoppelten, hochtemperaturtauglichen Sensors.

Ziel ist die Entwicklung einer Technologie zur batterie- und kabellosen Signalauswertung kapazitiver Sensorelemente für verschiedene Messgrößen. Die batterie- und kabellose Signalauswertung soll selbst bei extremen Umweltbedingungen die Auswertung der Sensoren gewährleisten. Die benötigte Signalverarbeitung wird in sicherer Entfernung platziert. Für viele Anwendungen in der Medizin-, Industrie- oder Kraftfahrzeugtechnik sind Messungen an Orten erforderlich, an denen eine elektrische Kontaktierung des Sensors nicht zweckmäßig oder nicht möglich ist. Bei der Messung in hermetisch abgeschlossenen Hohlräumen, an bewegten oder rotierenden Teilen und unter rauen Umgebungsbedingungen ist dies der Fall. Demzufolge muss ein vollständig passives Sensorelement eingesetzt werden. Mikroelektronische Schaltkreise, wie z.B. in der RFID -Technologie ermöglichen zwar eine Datenübertragung, können aber bei den geforderten hohen Temperaturen nicht verwendet werden, da diese zusätzlich noch den integrierten Schaltkreis zur Modulation enthalten.

\section{Induktive Kopplung}

Eine der wesentlichen Technologien zur batterie- und drahtlosen Signalübertragung ist der induktiv gekoppelte LC-Schwingkreis mit kapazitivem Sensor. Ohne weitere Schaltungskomponenten kann der LC-Schwingkreis mit kapazitiven oder induktiven Sensoren drahtlos ausgewertet werden. Entsprechende Sensorkonzepte zur drahtlosen Erfassung verschiedener Messgrößen sind bekannt. 
Die Resonanzfrequenz und die Güte der Sensor-Schwingkreise stellt dabei das Sensorsignal dar, welche drahtlos induktiv gekoppelt ermittelt werden.

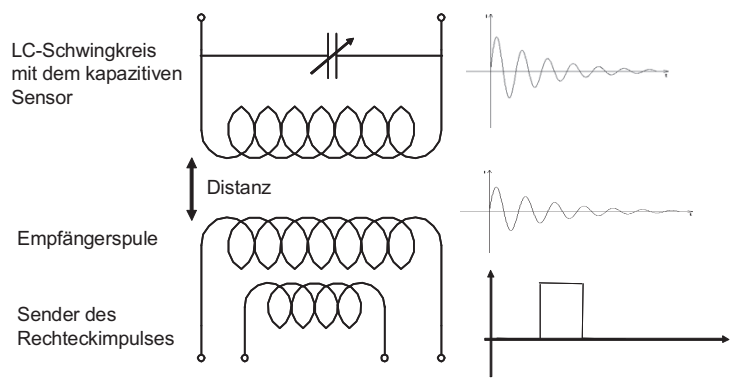

Abb. 1: Schematische Darstellung des induktiv gekoppelten Sensors

Die Abb. 1 zeigt eine schematische Darstellung des induktiv gekoppelten Sensors. Der Aufbau besteht aus drei Elementen. Den Anfang bildet der LC-Schwingkreis mit dem kapazitiven Sensor. Die Resonanzfrequenz wird durch das LC-Glied bestimmt. Die Induktivität $L$ ist konstant, die Kapazität $C$ von der Messgröße abhängig. Für die Dimensionierung des Schwingkreises sind die beiden unteren Beziehungen wesentlich.

Die Resonanzfrequenz

$f_{R}=\frac{1}{2 \pi} \sqrt{\frac{1}{L C}-\frac{R_{L}^{2}}{L^{2}}}$

und die Abklingkonstante

$\delta=\frac{R}{2 L}$.

Für eine gute Auswertbarkeit der Resonanzfrequenz ist eine kleine Abklingkonstante erforderlich, und damit ein geringer ohmscher Widerstand $R$. Ausgewertet wird die linear gedämpfte freie Schwingung des Schwingkreises. Angeregt wird der Schwingkreis mit einem Rechteckimpuls, übertragen von der Sendespule zur Induktivität des Schwingkreises. Die gedämpfte Schwingung wird von der Empfängerspule detektiert und von der Signalverarbeitung ausgewertet.

Für den Testaufbau wurde eine in LTCCTechnik realisierte Spule mit 2 mal 3 Windungen verwendet $(\mathrm{L}=0,16 \mu \mathrm{H}, \mathrm{R}=0,16 \mathrm{Ohm})$. Die Kapazität, des im Prototypen integrierten, von der Messgröße abhängigen, Kondensators deckt einen Bereich von 20 pF bis 40 pF ab. Damit ergibt sich ein Frequenzbereich von 25 $42 \mathrm{MHz}$.
Ein Rechteckimpuls mit möglichst steilem Anstieg wird durch die Sendespule geleitet (Abb. 2), dieser regt den LC-Schwingkreis mit Resonanzfrequenz an.

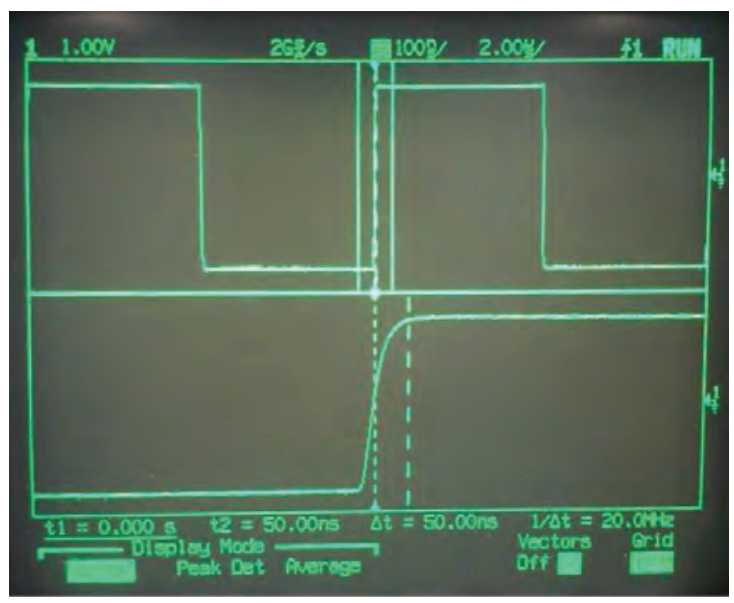

Abb. 2: Ausgang (Sender): Rechteckschwingung, $100 \mathrm{KHz}, 4 \mathrm{Vpp}$

Abb. 3 zeigt die Schwingung des LC-Schwingkreises und das empfangene Signal der Empfängerspule. Sende- und Empfängerspulen sind räumlich vom LC-Schwingkreis (Sensor) getrennt. In den Versuchen betrug die Distanz $5 \mathrm{~cm}$.

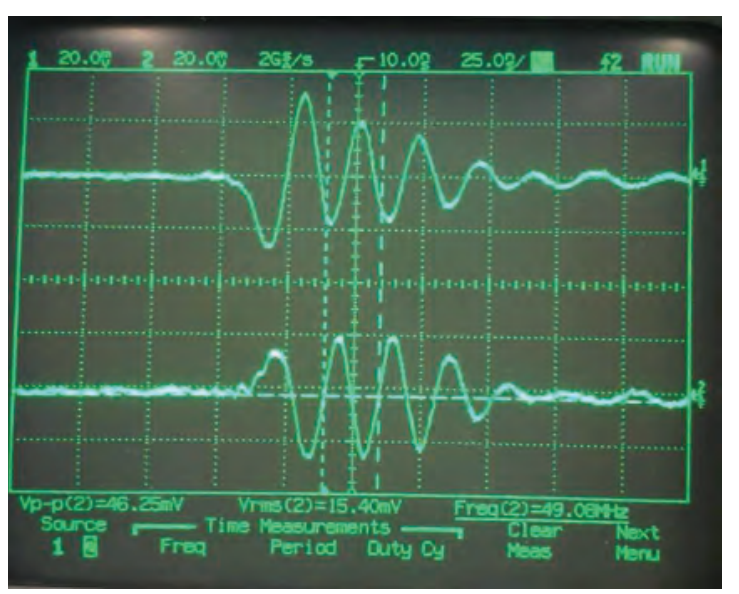

Abb. 3: Kondensator $=10 p F, 1 \perp$ Ausgang des LCSchwingkreis, $2 \perp$ Ausgang der Empfängerspule

\section{Aufbau eines Demonstrators}

Der verwendete Demonstrator besteht aus einer LTCC-Technik realisierten planaren Luftspule (Durchmesser $d_{L}=10 \mathrm{~mm}$ ), einer Sendespule $\left(d_{S}=20 \mathrm{~mm}\right)$ und einer Empfängerspule $\left(\mathrm{d}_{\mathrm{E}}=30 \mathrm{~mm}\right)$

Die Spule für den Schwingkreis ist hybrid integriert. Dies ermöglicht zum einen ein direktes Überprüfung der Sensorfunktion, zum anderen entfällt die geometrische Beschränkung für die Spule. Diese kann geometrisch größer gewählt 
werden, womit auch die Reichweite steigt. Aus Erfahrungswerten ist bekannt, dass die Reichweite einer rein passiven Anordnung ca. 1-2 mal dem Spulendurchmesser entspricht. In Abb. 4 ist die Lösung skizziert. Erst in einer weiteren Integrationsstufe soll die Induktivität in den Sensor integriert werden.

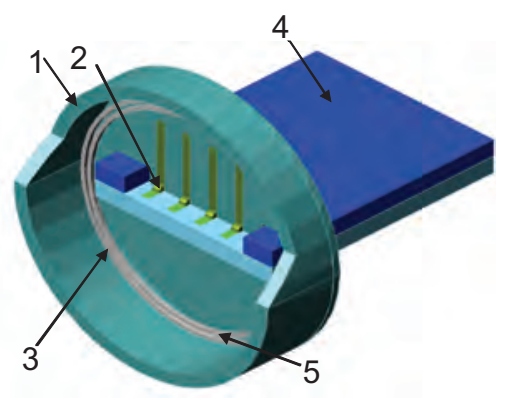

Abb. 4: Aufbau mit Spule (3), elektrische Verbindung von Spule und Sensor (2), Gehäuse (1) ohne Deckel, kapazitives Sensorelement (4), hermetisch dichtes Gehäuse für die Verbindung von Spule und Kondensator

Das Sensorsystem besteht aus der Induktivität (Luftspule) und dem kapazitiven Sensor. Beide Elemente sind elektrisch miteinander verbunden. Für die favorisierte Anwendung sind alle elektrischen Kontakte zu schützen, sie sollen nicht in Kontakt mit dem Messmedium gelangen. Um dies zu gewährleisten ist der Sensor derart aufgebaut, das die elektrischen Kontakte an der Stirnfläche nach außen geführt sind. Als Basismaterial wurde Silizium verwendet. Über eine entsprechende Öffnung im Membranchip können diese elektrisch kontaktiert werden. Im Spalt zwischen den Elektroden soll sich mindestens ein Feinvakuum befinden, so ist die Querempfindlichkeit aufgrund der Temperaturschwankung zu vernachlässigen. Das Feinvakuum wird durch einen Verschluss mit Glaslot erreicht. Die Sensorelemente sind mit MEMS-Technologien gefertigt.

Für die Induktivität wurde LTCC-Technologie verwendet. Der Sensorchip wird mit den stirnseitigen Kontakten in ein LTCC-Gehäuse gefügt. Für den rückseitigen Verschluss wird ebenfalls Glaslot verwendet. Das LTCCGehäuse beinhaltet die zum Schwingkreis gehörige Induktivität, ausgeführt als Luftspule.

In der Abb. 5 sind die reale Spule und der reale Sensor im Einzelnen abgebildet.
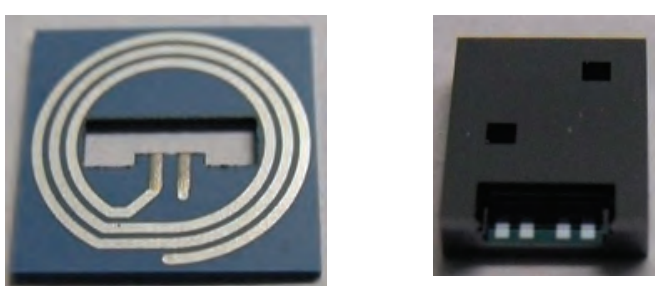

Abb. 5: Links, Spule in LTCC $\left(d_{L}=10 \mathrm{~mm}, L=0,16\right.$ $\mu H, R=0,16 \mathrm{Ohm})$, rechts, kapazitives Sensorelement $\left(3 \mathrm{~mm}^{*} 4 \mathrm{~mm}\right)$

\section{Evaluierung des Sensors}

Für die Evaluierung des Sensorsystems mit induktiver Kopplung wurden zwei Sensorelemente verwendet, ein kapazitiver Leitfähigkeitssensor und ein kapazitiver Drucksensor. Die Ergebnisse sind in Abb. 6 und 7 dargestellt.

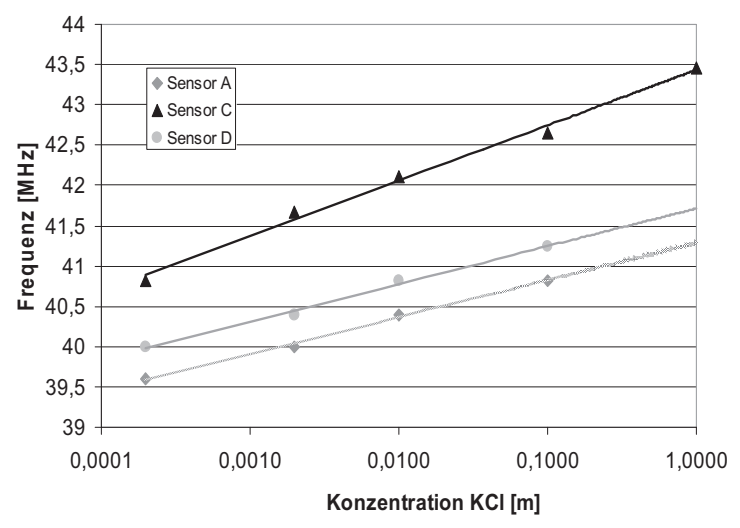

Abb. 6: Kapazitiver Sensor zur Messung der Konzentration über die Leitfähigkeit

Abb. 6 zeigt die Auswertung, es wurden Sensorelemente für verschieden Messbereiche verwendet. Der genaue Aufbau des Sensors ist für Versuche nicht entscheidend. Die Grundkapazität liegt bei ca. 30 pF. Bei den Messungen betrug der Abstand zwischen Sensor und Sende- und Empfängerspule $5 \mathrm{~cm}$. Die Messwerte zeigen eine gute Auswertbarkeit. Zur Bestimmung der Frequenz wurde ein Oszilloskop verwendet. Die Frequenzmessung ist damit noch Fehlerbehaftet, eine ausreichende Statistik war noch nicht möglich. Die Frequenz der Rechteckschwingung zur Anregung betrug $100 \mathrm{kHz}$, so konnte, bei einer Abtastrate von $100 \mathrm{~Hz}$, leicht ein Mittelwert aus 1000 Einzelmessungen verwendet werden. Eine entsprechende Signalauswertung wird noch entwickelt.

Als zweites Sensorelement wurde ein kapazitiver Drucksensor (Abb. 7) verwendet. Auch hier zeigt sich ein auswertbares Messsignal. Leider 
können noch keine statistischen Angaben über die Genauigkeit des Sensors geliefert werden.

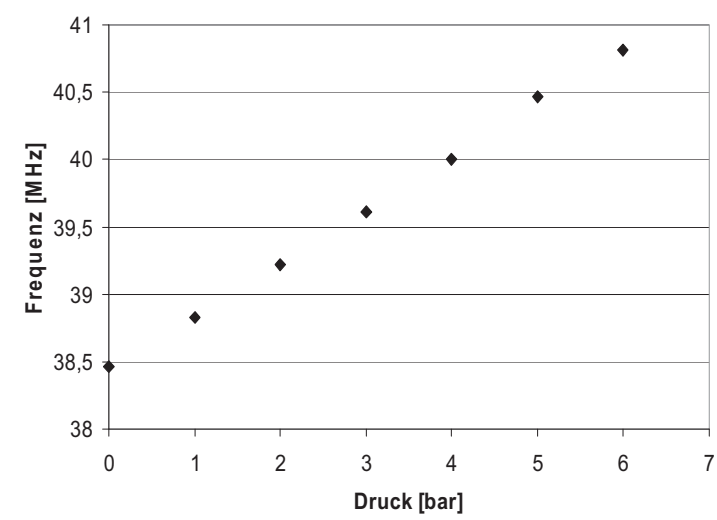

Abb. 7: Kapazitiver Membran-Drucksensors, Frequenz in Abhängigkeit der Messgröße (Druck)

\section{Zusammenfassung und Ausblick}

Es wurde ein System zur drahtlosen Anbindung kapazitiver Sensoren durch induktiv gekoppelte Felder vorgestellt. Durch den Verzicht mikroelektronischer Komponenten im LCSchwingkreis, ist dieser Aufbau für harsche Umgebungsbedingungen geeignet.

Es werden weitere kapazitive Sensoren für dieses System evaluiert und eine Signalauswertung für die Frequenzmessung aufgebaut. 